\title{
Traditional Chinese Medicine Constitution Correlated with Ischemic Stroke: A Systematic Review and Meta-Analysis
}

\author{
Tianyi Zhang $\mathbb{D}^{1},{ }^{1}$ Hui Luo, ${ }^{2}$ Dawei Wei $\mathbb{D},{ }^{1}$ Xiaolong Xie $\mathbb{D},{ }^{1}$ Cinyu Yang $\mathbb{D},{ }^{1}$ \\ Bowen Liu $\mathbb{D}^{1}$, and Ying Gao $\mathbb{D}^{1}$ \\ ${ }^{1}$ Dongzhimen Hospital, Beijing University of Chinese Medicine, 5 Haiyuncang, Dongcheng District, Beijing 100700, China \\ ${ }^{2}$ Institute for Tibetan Medicine, China Tibetology Research Center, Beijing 100101, China \\ Correspondence should be addressed to Ying Gao; gaoying973@126.com
}

Received 23 February 2021; Revised 24 April 2021; Accepted 11 June 2021; Published 25 June 2021

Academic Editor: Bhushan Patwardhan

Copyright (C) 2021 Tianyi Zhang et al. This is an open access article distributed under the Creative Commons Attribution License, which permits unrestricted use, distribution, and reproduction in any medium, provided the original work is properly cited.

Objective. To investigate the correlation between traditional Chinese medicine (TCM) body constitution and ischemic stroke (IS). Methods. Literature search was conducted in databases including Wanfang database, Chongqing VIP, China National Knowledge Infrastructure, Embase, and PubMed from inception to November 16, 2020. Observational studies evaluating the association between TCM body constitution and IS were included for analysis. The distribution of body constitutional types in IS patients was pooled into meta-analysis. The correlation between constitution and IS was presented by the odds ratio (OR) and 95\% CI through the comparison between IS and the general population. Results. 41 studies involving 11,211 participants were included. Among the nine constitution types, qi-deficiency constitution (QDC), phlegm-dampness constitution (PDC), and blood stasis constitution (BSC) are the common types, accounting for 25\% [0.22, 0.29], 23\% [0.20, 0.29], and 17\% [0.13, 0.22], respectively, in IS patients. The proportion of PDC and QDC among IS patients is 2.34 times and 3.47 times higher than that in the general population, respectively (OR and 95\% CI: $2.34[1.39,3.94], 3.47[1.61,7.50]$, respectively). Conclusion. PDC and QDC are the common constitutions in IS patients and may have a potential correlation with the incidence of IS. Due to the low or moderate quality of included studies, more well-designed prospective studies are warranted to further evaluate the relationship between TCM constitutions and IS.

\section{Introduction}

Stroke is the second most mortal disease globally. Ischemic stroke, a sudden functional loss caused by the cut-off of the bloodstream to the brain, is the most common type that accounts for over $85 \%$ of all cases [1]. Every year, about 6 million people die from stroke globally, and even worse, the illness, disability, and premature death caused by stroke will double by 2035 [2]. The recurrence rate within a decade is as high as $39.2 \%$ [3-5]. On the one hand, the treatment and rehabilitation of stroke will bring huge economic burden to the society, but on the other hand the current therapeutic approaches are insufficient for reducing deaths caused by stroke. Prevention of stroke is of great significance to the family and the society. Risk factor management is important for preventing stroke. In studies focusing on stroke risk factors, the Framingham Stroke Scale is currently the most widely used stroke risk assessment tool in western countries [6]. However, it overestimated the risk of stroke in Chinese [7]. Besides, these studies focus on defining risk factors of stroke, without providing a clear definition of the high-risk group of stroke [8].

In traditional Chinese medicine, preventative treatment before the disease onset has been practiced for thousands of years. TCM theory states that Chinese medicine has a great advantage in the preventive treatment of disease. TCM constitution identification is the effective approach of "treating disease before onset." [9] The TCM constitution is a relatively stable and integrated intrinsic feature of physiological function, morphological structure, and psychological state formed by innate endowment and acquired disposition. The TCM constitution can be classified into nine 
basic types: yang-deficiency constitution (YADC), qi-deficiency constitution (QDC), qi stagnation constitution (QSC), phlegm-dampness constitution (PDC), yin-deficiency constitution (YIDC), damp-heat constitution (DHC), blood stasis constitution (BSC), balance constitution (BC), and inherited special constitution (ISC). Among these, $\mathrm{BC}$ is normal constitution and all the others are biased constitutions. People with different constitution have different characteristics in physiological manifestation, pathological state and morbidity tendency, and so on. For instance, the $\mathrm{BC}$, formed by the harmony of yin, yang, qi, and blood, is characterized by ruddy complexion, vigorous energy, and moderate posture. People with BC can adapt to the environment easily and are unlikely to suffer from diseases.

There are emerging clinical studies focusing on TCM constitution and its correlation with IS. These studies provide a basis for investigating the clinical distribution of TCM constitution in IS patients. However, no systematic review is found among existing studies. Thus, a summary is needed to incorporate the existing literature. To provide solid evidence for clinical and scientific research based on larger sample, a meta-analysis is conducted on the above studies.

\section{Methods}

2.1. Registration. The protocol was registered and published at PROSPERO (CRD42020209602), accessible at https:// www.crd.york.ac.uk/PROSPERO/display_record.php? RecordID=209602.

\subsection{Searching Strategy}

2.2.1. Literature Search. We collected clinical literatures on the association between TCM constitution and IS from databases such as Wanfang Database, Chongqing VIP, China National Knowledge Infrastructure, Embase, and PubMed. The literature is either in English or in Chinese. The dates range from the day when the database was founded to November 16, 2020. The keywords are "ischemic stroke," "constitution," and "Chinese medicine." For Chinese databases, searching strategies are "ischemic stroke+ constitution" in title or abstract, and "Chinese medicine" in full text. For English databases, search strategies are ((ischemic stroke) AND constitution AND (TCM OR Chinese medicine)).

\subsection{Eligibility Criteria}

2.3.1. Inclusion. (1) All clinical studies (including crosssectional studies, case-control studies, and cohort studies) in Chinese or English on TCM constitution and ischemic stroke correlation are included. (2) All patients in studies should be clearly diagnosed with ischemic stroke. (3) TCM constitution recognition: TCM constitution is recognized by "classification and determination of TCM constitution" norm report by China Correlation of Chinese Medicine (CACM) in 2009 [10].
2.3.2. Exclusion. (1) The basic characteristic of patients are not reported (gender, age etc.). (2) The results' data reported are deficient. 3. Research reports share the same patient samples.

2.4. Literature Screening and Data Extraction. All papers are first screened by title/abstract in line with the eligibility criteria on NoteExpress software. Those that pass the initial screen are checked throughout. Then, only those that pass the two screenings are used for data extraction. Each paper is screened and extracted by two investigators independently, and the results are cross-checked in every step. In the case of divergence in cross-check, the checked paper will be examined by the $3 r d$ investigator for the final decision. The main extract data include researcher's name, study type, period and region, participant source, sample size, age, gender, constitution results, and measures of quality control.

2.5. Quality Evaluation. The Unit States Agency for Healthcare Research (AHRQ) publishes standards to evaluate cross-sectional study [11], including 11 items with the highest mark of 11 . The items include observation period, eligibility criteria, research object continuity, data source, and quality control. As for the mark, $0-3$ is considered as low quality, $4-7$ as medium quality, and $8-11$ as high quality. The Newcastle-Ottawa scale (NOS) recommends case-control study and cohort study [12], containing 11 items in 3 prospects. The NOS mainly evaluates the comparability between groups, population selection, and evaluation of exposure factors. Its highest mark is 9, and any mark over 6 is considered as high quality.

2.6. Statistical Analysis. Two meta-analysis sets are constructed based on the 41 studies included.

2.6.1. Analysis Dataset 1. The analysis dataset 1 is a metaanalysis of the association between TCM constitution and IS. This part aims to explore the risk TCM constitution in IS. The meta-analysis is conducted on the studies that report distribution of TCM constitution in both IS patients and general population. ReviewManager (version 5.3) software is used for the meta-analysis. The effect values are described by odds ratio (OR) and $95 \%$ confidence interval ( $95 \% \mathrm{CI}) . I^{2}$ is defined as the heterogeneity of the meta-analysis. If the heterogeneity is low $\left(I^{2} \leq 25 \%\right)$, the fix effect model will be chosen; if the heterogeneity is high $\left(25 \%<I^{2}<75 \%\right)$, the random effect model will be chosen; if the heterogeneity is much higher $\left(I^{2} \geq 75 \%\right)$, qualitative description will replace meta-analysis.

2.6.2. Analysis Dataset 2. The analysis dataset 2 is a metaanalysis of the distribution of TCM constitution in IS patients. This part aims to explore the total rate of different TCM constitution types in patients. The meta-analysis is conducted on the studies that report distribution of different constitution types in IS patients. The data of studies in analysis 2 is featured with that they only provide the number 
of people and events in one group. At present, ReviewManager, a commonly used meta-analysis software, cannot achieve single-rate meta-analysis. In this analysis, $\mathrm{R}$ version 3.2.2 is used for this meta-analysis. The results are shown by total ratio of different TCM constitution types in IS population and its 95\% CI. When the heterogeneity is much higher, the subgroup analysis will be conducted according to region, gender, and related factors.

\section{Results}

3.1. Literature Search. Following the searching strategy, we collected a total of 932 papers, with 41 passing the eligibility criteria. The paper includes 11,211 eligible subjects. The flow diagram and results of literature searching are shown in Figure 1 [13-53]. One of the studies is reported in English and the rest are in Chinese. Table 1 shows the fundamental features of the studies.

3.1.1. Meta-Analysis 1: Meta-Analysis of the Distribution of TCM Constitution in IS and the General Population. QDC. In total, 5 articles investigate the QDC's distribution in IS and the general population [17, 21, 32, 44, 50]. Heterogeneity is tested among the studies $\left(I^{2}=68 \%, P=.01\right)$, and the random-effects model is implemented. The meta-analysis reveals that $\mathrm{OR}=2.34,95 \% \mathrm{CI}$ is $[1.39,3.94]$ and finds statistically significant differences $(P=.001)$. The results are shown in Figure 2.

PDC. In total, 6 articles report PDC's distribution in IS and the general population $[17,21,26,32,44,50]$. Heterogeneity is tested among the studies $\left(I^{2}=73 \%, P=.002\right)$ and randomeffects model is used. Meta-analysis reveals that $\mathrm{OR}=3.47$, 95\% CI $[1.61,7.50]$, indicating statistically significant differences $(P=0.002)$. The results are shown in Figure 3 .

Other TCM Constitutions. In total, 5 articles report blood stasis constitution's distribution in IS and the general population $[18,22,33,45,51]$. And 4 report yang-deficiency constitution (YADC), yin-deficiency constitution (YIDC), damp-heat constitution (DHC), qi stagnation constitution (QSC), balance constitution (BC), and inherited special constitution (ISC) $[18,22,45,51]$. The meta-analysis reveals that the difference is not statistically significant $(P>.05)$. The results are listed in Table 2.

3.1.2. Meta-Analysis 2: Meta-Analysis of the Distribution of TCM Constitutions in IS Patients. The meta-analysis is conducted on each TCM constitution type of IS patients report as an index. Among the 41 studies, 1 case-control study of PDC only covers the proportion of PDC, and the other 40 studies report data on the proportion of various types of TCM constitution. Therefore, 41 studies are included in the meta-analysis of PDC. The results show that these studies are highly heterogenous. Considering the particularity of the cross-sectional study in the field of TCM constitution (see Section 4.3), the random effect model is used for meta-analysis. PDC, QDC, and blood stasis constitution (BSC) accounts for more than 15\% of all. The results of meta-analysis are shown in a forest map, and the rest of the TCM constitutions less than $15 \%$ are listed in a table.

QDC. In total, 40 articles report QDC's distribution in IS patients $[13-25,27-53]$. Heterogeneity is tested among the studies $\left(I^{2}=93 \%, P<.01\right)$ and random-effects model is used. Meta-analysis finds the rate $=25 \%$ and $95 \%$ CI is $[0.22,0.29]$. The results are shown in Figure 4.

PDC. In total, 41 articles report PDC's distribution in IS patients [13-53]. Heterogeneity is tested among all studies $\left(I^{2}=96 \%, P<0.01\right)$ and random-effects model is used. Metaanalysis finds that rate $=23 \%$ and $95 \%$ CI is $[0.20,0.29]$. The results are shown in Figure 5.

BSC. In total, 40 articles report blood stasis constitution's distribution in IS patients [13-53]. Heterogeneity is tested out among the studies $\left(I^{2}=98 \%, P<0.01\right)$ and randomeffects model is used. Meta-analysis finds that rate $=17 \%$ and $95 \%$ CI is $[0.13,0.22]$. The results are shown in Figure 6.

Other TCM Constitutions. In total, 40 articles that report YADC, YIDC, DHC, QSC, BC, and ISC's distribution in IS patients [13-53]. Meta-analysis finds the rate is lower than $15 \%$. The detailed results are shown in Table 3.

3.1.3. Subgroup Analysis: Meta-Analysis of TCM Constitution's Distribution in Different Areas and Genders. Different Areas. In total, 7 articles report the distribution of constitution in IS patients in north China $[14,16,21,25,37,46,48]$. Meta-analysis is shown by rate [95\% CI]. It finds PDC accounts for 33\% [0.20-0.50], QDC for $28 \%$ [0.20-0.39], and BSC for 27\% [0.18-0.38]. In total, 16 articles report the distribution of constitution in east China $[15,17-20,23,24,26,29,33,35,36,41,42,51]$. It finds that PDC accounts for 18\% [0.15-0.21], QDC for $25 \%$ [0.22-0.29], and BSC for 9\% [0.06-0.13]. And 10 articles report the distribution of constitution in central south China $[13,23,28,31,32,34,38,44,45,49]$. It finds that PDC accounts for $25 \%$ [0.18-0.33], QDC for 20\% [0.16-0.26], and BSC for $17 \%$ [0.10-0.28]. A total of 5 articles report the distribution of constitution in northeast China $[27,30,39,43,47]$. It finds that PDC accounts for $38 \%$ [0.17-0.65], QDC for 24\% [0.16-0.34], and BSC for $36 \%$ [0.21-0.53].

Different Genders. In total, 10 articles report the specific data of constitutions types by gender $[15,20,21,24,25,32,37,45,48,51]$. No gender difference is found in the distribution of PDC in IS patients (OR: 1.09, 95\% CI: $0.72-1.65 P=.68$ ), in that of QDC (OR: $1.18,95 \% \mathrm{CI}$ : $0.77-1.81, P=0.46$ ), and neither in that of BSC (OR: 0.78 , 95\% CI: $0.42-1.46, P=.43)$.

\section{Discussion}

4.1. Analysis of TCM Constitution Types in IS and the General Population. The meta-analysis based on 11,211 samples 


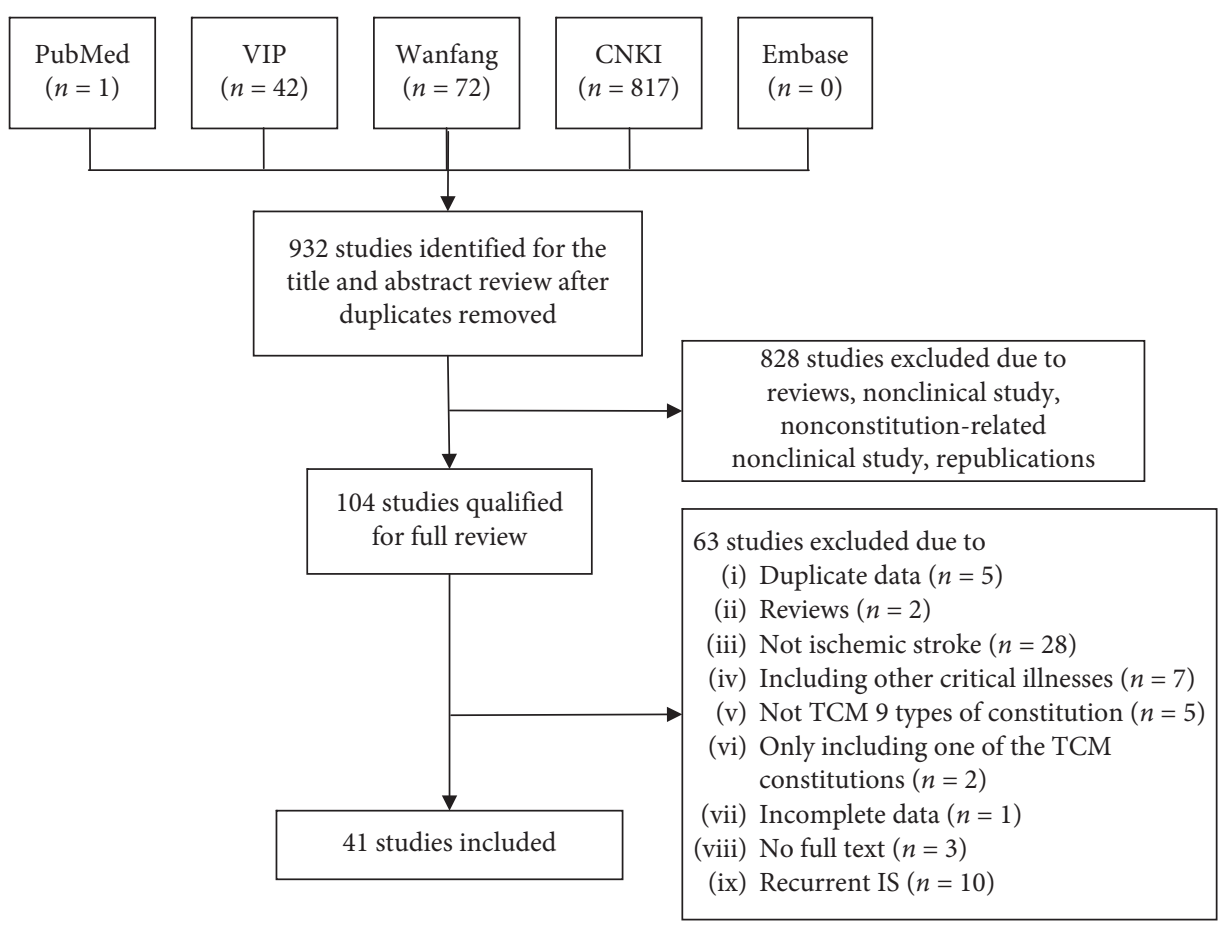

Figure 1: Flow diagram and results of literature searching.

finds PDC, QDC, and BSC, respectively, account for (rate and $95 \%$ CI: $23 \%[0.20,0.29], 25 \%[0.22,0.29]$ and $17 \%$ [0.13, $0.22]$ ) of all patients. The one based on 4,608 samples finds the proportion of PDC and QDC in IS patients is 2.34 and 3.47 times higher than that in the general population, respectively (OR and 95\% CI: 2.34 [1.39, 3.94], 3.47 [1.61, $7.50]$ ), with statistically significant differences. Based these findings, the study suggests PDC, QDC, and BSC are the main TCM constitution of IS patients, QDC and PDC constitution may be the risky factors of IS, and PDC is the most common one.

The distribution of TCM constitution types in IS patients is quite different from that in the general population. Professor Wang Qi conducted a cross-sectional field study on the distribution of TCM constitution, which includes 21,948 cases from 9 provinces and cities during 2005-2007 [54]. The results find that $\mathrm{BC}$ tops all, accounting for 32.14\% among all. And QDC, DHC, and YADC are ranked in the top three in terms of biased constitutions, accounting for $13.42 \%, 9.08 \%$, and $9.04 \%$, respectively. In this meta-analysis, QDC has the highest proportion (25\%) in all TCM constitution types, close to the proportion of $\mathrm{BC}$ in the general population, much higher than the proportion of QDC in the general population (13.42\%). Similarly, PDC and BSC are like so. The BC only accounts for $7 \%$. Therefore, it is advised to identify the constitution before the onset of the disease, and health education is an effective way to prevent the high-risk group from turning into bias constitution such as QDC, PDC, and BSC. In addition, identifying the bias constitution earlier could promote the return of bias constitution to $\mathrm{BC}$ through TCM therapies, which is of great significance to prevent the occurrence of IS.
In terms of the regional distribution proportion of TCM constitutions, the PDC and BSC occupy the highest proportions in Northeast China, followed by North China, Central South China, and East China. The QDC is most common in North China, followed by East China, Northeast China, and Central South China. The difference in distribution among regions is related to the climate, water, and dietary habit. TCM emphasizes the harmony between people and nature. So, this study may be closely related to climatic and dietary factors. The environment in North and Northeast China is colder and drier than the East and Central South China, and people in North and Northeast China prefer salty food and spirit. So, PDC and BSC have higher proportion there, while the climate in North and East China is warmer but not hot, moister but not humid. Besides, the food is light in these regions. So the proportion of QDC there is higher than that in the Northeast and the Central south China. Further studies need to be done to explore other reasons.

In terms of the proportion of gender distribution of TCM constitution, the proportion of PDC, QDC, and BSC is the same in males and females in this study. This is possibly because QDC, PDC, and BSC are the main constitution of IS, and the pathological mechanism results in the same constitution regardless of gender.

4.2. Methodological Problems in the Studies of TCM Constitution in IS Patients. This study discovers many methodological problems through quality evaluation. The international reporting standard for observational studies statement (Strengthening the Reporting of Observational Studies in Epidemiology, STROBE) has not been applied for 
TABLE 1: The basic characteristics and quality score of studies included.

\begin{tabular}{|c|c|c|c|c|c|c|c|c|}
\hline Study ID & $\begin{array}{l}\text { Study } \\
\text { type }\end{array}$ & Area & Participant & $\begin{array}{l}\text { Sample } \\
\text { size }\end{array}$ & Age & $\begin{array}{c}\text { Gender } \\
\text { ratio }(\mathrm{M} / \mathrm{F})\end{array}$ & Constitution & $\begin{array}{l}\text { Quality } \\
\text { score }\end{array}$ \\
\hline Lijin [13] & CS & Guangdong & Hospital & 183 & $67.8 \pm 11.2$ & $107 / 76$ & 9 types & 6 \\
\hline Sunshuai [14] & CS & Beijing & Hospital & 61 & $64.13 \pm 7.525$ & $45 / 16$ & 9 types & 8 \\
\hline Zhangxiu [15] & CS & Fujian & Hospital & 150 & $70.4 \pm 11.2$ & $87 / 63$ & 9 types & 6 \\
\hline Liuxia [16] & CS & Beijing & Hospital & 125 & $65.69 \pm 11.54$ & $79 / 46$ & $\begin{array}{c}8 \text { types (without } \\
\text { Balanced) }\end{array}$ & 5 \\
\hline Luyong [17] & CS & Shanghai & Hospital & 200 & $70.64 \pm 4.33$ & $102 / 98$ & $\begin{array}{l}8 \text { types (without } \\
\text { inherited special) }\end{array}$ & 7 \\
\hline Mawen [18] & CS & Shandong & Hospital & 98 & NI & $53 / 55$ & 9 types & 5 \\
\hline Songwei [19] & CS & Shandong & Hospital & 127 & $69 \pm 18$ & $78 / 49$ & 9 types & 6 \\
\hline Lixiu [20] & CS & Shandong & Hospital & 523 & $66.87 \pm 10.26$ & $325 / 198$ & 9 types & 9 \\
\hline Liyu [21] & CS & Beijing & Hospital & 227 & $73.51 \pm 8.19$ & $100 / 78$ & 9 types & 7 \\
\hline Liangmin [22] & CS & Guangdong & Hospital & 150 & $68.08 \pm 12.15$ & $83 / 67$ & 9 types & 8 \\
\hline Maxian [23] & CS & Jiangsu & Hospital & 299 & NI & $178 / 121$ & 9 types & 8 \\
\hline Wuhong [24] & CS & Shandong & Hospital & 403 & NI & $254 / 149$ & 9 types & 6 \\
\hline Xuyin [25] & CS & Beijing & Hospital & 500 & $62.3 \pm 11.9$ & $325 / 175$ & 9 types & 8 \\
\hline Zhenxiao [26] & CS & Shanghai & $\begin{array}{c}\text { Community \& } \\
\text { hospital }\end{array}$ & 475 & NI & $232 / 243$ & Phlegm-dampness & 8 \\
\hline Jiaai [27] & $\mathrm{CC}$ & Liaoning & Hospital & 510 & NI & $261 / 249$ & 9 types & 7 \\
\hline Lili [28] & CS & Guangdong & Hospital & 276 & $65.95 \pm 11.03$ & $187 / 89$ & 9 types & 9 \\
\hline $\begin{array}{l}\text { Zhuangshan } \\
\text { [29] }\end{array}$ & CS & Shandong & Hospital & 210 & $64.43 \pm 9.58$ & $114 / 96$ & 9 types & 8 \\
\hline Jiaai [30] & CS & Liaoning & Hospital & 620 & NI & $315 / 305$ & 9 types & 9 \\
\hline Zhumei [31] & CS & Guangxi & Hospital & 90 & 68 & $53 / 37$ & 9 types & 8 \\
\hline Wangkai [32] & CS & Hubei & Hospital & 156 & $72.56 \pm 8.12$ & $40 / 38$ & 9 types & 9 \\
\hline $\begin{array}{l}\text { Wangqun } \\
\text { [33] }\end{array}$ & CS & Shandong & Hospital & 142 & $67.52 \pm 10.62$ & $78 / 64$ & 9 types & 7 \\
\hline Lizheng [34] & CS & Henan & Hospital & 110 & $59.45 \pm 11.44$ & $64 / 46$ & 9 types & 8 \\
\hline Zhanghai [35] & Cohort & Shanghai & Hospital & 168 & $70.14 \pm 11.4$ & $101 / 67$ & 9 types & 8 \\
\hline Liangkun [36] & CS & Shandong & Hospital & 100 & NI & NI & 9 types & 6 \\
\hline Mengshu [38] & Cohort & Guangdong & Hospital & 803 & NI & $531 / 272$ & 9 types & 9 \\
\hline Fengpeng [39] & CS & Jilin & Hospital & 202 & $64.23 \pm 10.04$ & $116 / 86$ & 9 types & 7 \\
\hline Cuisai [41] & CS & Shandong & Hospital & 153 & $64.05 \pm 11.84$ & $99 / 54$ & 9 types & 8 \\
\hline $\begin{array}{l}\text { Huangling } \\
\text { [42] }\end{array}$ & CS & Jiangxi & Hospital & 180 & $66.33 \pm 9.28$ & $105 / 75$ & 9 types & 7 \\
\hline Fuxin [43] & CS & Jilin & Hospital & 176 & $63.56 \pm 10.41$ & $112 / 64$ & 9 types & 6 \\
\hline Lili [47] & CS & Jilin & Hospital & 248 & $63.40 \pm 10.38$ & $166 / 82$ & 9 types & 7 \\
\hline Jichuan [49] & CS & Guangdong & Hospital & 100 & $49.61 \pm 4.85$ & $61 / 39$ & 9 types & 6 \\
\hline $\begin{array}{l}\text { Gaocheng } \\
\text { [52] }\end{array}$ & CS & Shandong & Hospital & 62 & NI & $36 / 26$ & 9 types & 6 \\
\hline Gaoyang [53] & CS & $\begin{array}{l}\text { Beijing, Guangdong, } \\
\text { Henan, Jilin, Shanxi }\end{array}$ & Hospital & 1741 & $62.7 \pm 9.63$ & $1106 / 635$ & 9 types & 7 \\
\hline Wanghua [37] & CS & Hebei & Hospital & 198 & NI & $126 / 72$ & 9 types & 8 \\
\hline Xieqing [40] & CS & Yunnan & Hospital & 52 & NI & $40 / 12$ & 9 types & 8 \\
\hline Zouzhi [44] & CC & Guangdong & Hospital & 40 & $71.27 \pm 15.34$ & $18 / 22$ & 9 types & 4 \\
\hline Zhouhan [45] & CS & Hunan & Hospital & 303 & $64.87 \pm 11.19$ & $175 / 128$ & 9 types & 9 \\
\hline $\begin{array}{l}\text { Wangfang } \\
{[46]}\end{array}$ & Cohort & Beijing, Shanxi & Hospital & 274 & $62.49 \pm 8.63$ & $188 / 86$ & 9 types & 7 \\
\hline Wuxiao [48] & CS & Beijing & Community & 400 & NI & $249 / 151$ & 9 types & 9 \\
\hline Liu et al. [50] & CS & Shanghai & Community & 124 & $69.33 \pm 7.53$ & $66 / 58$ & 9 types & 8 \\
\hline Yuhong [51] & CS & Shandong & Hospital & 252 & $65.12 \pm 12.30$ & $145 / 107$ & 9 types & 8 \\
\hline
\end{tabular}

CS: cross-sectional study; CC: case-control study; NI: no information.

format reports in any research included [55]. The reporting rate of each item is pretty low according to the evaluation standard of AHRQ cross-sectional study. Very few studies report the quality control measures clearly, which may lead to bias in the research results. The studies are highly heterogenous ( $\mathrm{I}^{2}$ value is larger) from the meta-analysis of TCM constitution distribution.
From the perspective of research design, these studies do not contain enough basic information, including but not limited to the time range, the approach to including subjects, the methodological details of constitution identification, nationality, age, gender, body mass index, blood glucose, blood lipids, blood pressure, rejection rate, and funding status. In the statistical analysis of data, some studies omit 


\begin{tabular}{|c|c|c|c|c|c|c|c|c|c|}
\hline \multirow[b]{2}{*}{ Study or subgroup } & \multicolumn{2}{|c|}{ Experimental } & \multicolumn{2}{|c|}{ Control } & \multirow[b]{2}{*}{ Weight (\%) } & \multirow{2}{*}{$\begin{array}{c}\text { Odds ratio } \\
\mathrm{M}-\mathrm{H} \text {, random, } 95 \% \mathrm{CI}\end{array}$} & \multirow{2}{*}{\multicolumn{2}{|c|}{$\begin{array}{c}\text { Odds ratio } \\
\mathrm{M}-\mathrm{H} \text {, random, } 95 \% \mathrm{CI}\end{array}$}} & \\
\hline & Events & Total & Events & Total & & & & & \\
\hline Liujia et al. [50] & 47 & 124 & 1106 & 3624 & 27.6 & $1.39[0.96,2.01]$ & & 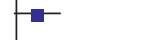 & \\
\hline Liyu et al. [21] & 62 & 128 & 7 & 49 & 16.9 & $5.64[2.36,13.48]$ & & & \\
\hline Luyong et al. [17] & 63 & 200 & 25 & 200 & 24.4 & $3.22[1.92,5.38]$ & & $\longrightarrow-$ & \\
\hline Wangkai [32] & 30 & 78 & 20 & 78 & 20.7 & $1.81[0.92,3.59]$ & & - & \\
\hline Zouzhi et al. [44] & 7 & 40 & 4 & 37 & 10.5 & $1.75[0.47,6.55]$ & & " & \\
\hline \multirow{2}{*}{$\begin{array}{l}\text { Total }(95 \% \mathrm{CI}) \\
\text { Total events }\end{array}$} & & 570 & & 3988 & 100.0 & $2.34[1.39,3.94]$ & & & \\
\hline & 209 & & 1162 & & & & & & \\
\hline \multirow{2}{*}{\multicolumn{6}{|c|}{$\begin{array}{l}\text { Heterogeneity: } \text { tau }^{2}=0.22 ; \text { chi }^{2}=12.63, \mathrm{df}=4(P=0.01) ; I^{2}=68 \% \\
\text { Test for overall effect: } Z=3.20(P=0.001)\end{array}$}} & 0.01 & 0.1 & 1 & 10 \\
\hline & & & & & & & Favours (control) & Favours (e & experimental) \\
\hline
\end{tabular}

FIGURE 2: Meta-analysis of QDC's distribution in IS and the general population.

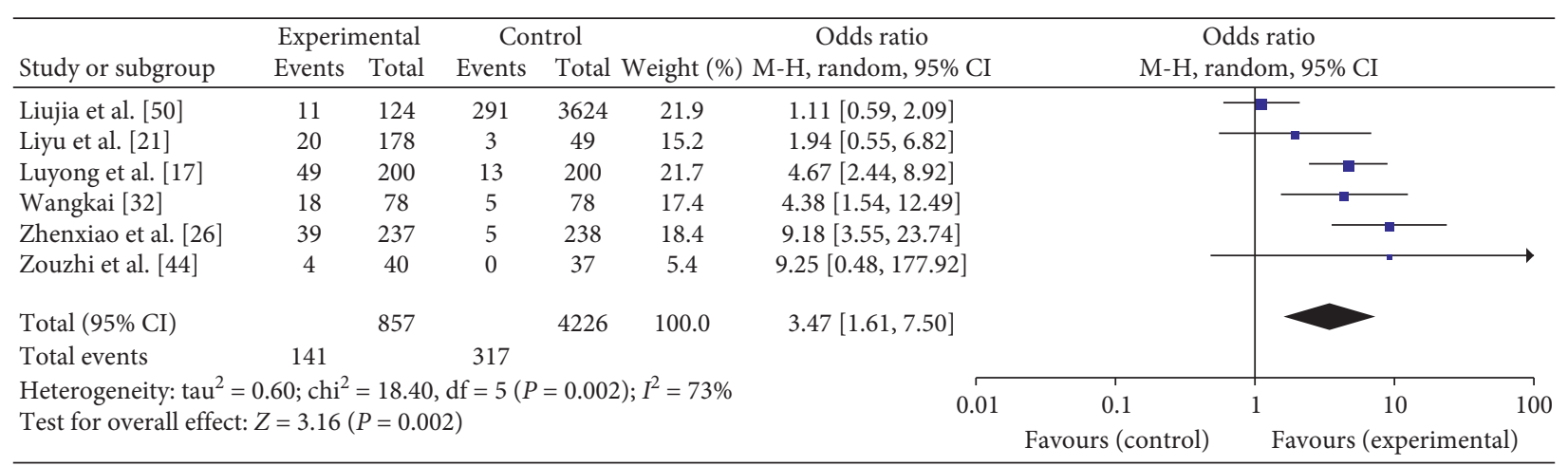

FIgURE 3: Meta-analysis of PDC's distribution in IS and the general population.

TABLE 2: Meta-analysis of other TCM constitutions' distribution in IS and the general population.

\begin{tabular}{|c|c|c|c|c|c|c|c|c|}
\hline \multirow{2}{*}{ TCM constitution } & \multicolumn{2}{|c|}{ Event/total number } & \multicolumn{2}{|c|}{ Heterogeneity test } & \multirow{2}{*}{ OR } & \multirow{2}{*}{$95 \% \mathrm{CI}$} & \multirow{2}{*}{$Z$} & \multirow{2}{*}{$P$} \\
\hline & IS population & General population & $I^{2}(\%)$ & $P$ & & & & \\
\hline Blood stasis constitution (BSC) & $93 / 620$ & $105 / 3988$ & 5 & 0.38 & 1.44 & $0.91-2.27$ & 1.57 & 0.12 \\
\hline Yang-deficiency constitution (YADC) & $82 / 542$ & $883 / 3910$ & 79 & 0.002 & 1.29 & $0.56-3.00$ & 0.60 & 0.55 \\
\hline Yin-deficiency constitution (YIDC) & $88 / 542$ & $352 / 3910$ & 41 & 0.17 & 1.19 & $0.69-2.05$ & 0.63 & 0.53 \\
\hline Damp-heat constitution (DHC) & $75 / 542$ & $1218 / 3910$ & 66 & 0.03 & 0.87 & $0.39-1.92$ & 0.35 & 0.73 \\
\hline Qi stagnation constitution (QSC) & $21 / 542$ & $64 / 3910$ & 16 & 0.31 & 1.01 & $0.42-2.42$ & 0.01 & 0.99 \\
\hline Balance constitution (BC) & $110 / 542$ & $1311 / 3910$ & 95 & $<0.00001$ & 0.37 & $0.10-1.38$ & 1.48 & 0.14 \\
\hline Inherited special constitution (ISC) & $3 / 420$ & $85 / 3788$ & 0 & 0.78 & 0.53 & $0.16-1.71$ & 1.06 & 0.29 \\
\hline
\end{tabular}

the correlation between TCM constitutions and gender, age, disease degree, and other related factors. The individual studies find three or more types of mix constitution, or indicate the proportion of balanced constitution in the subjects is 0 . This is because the researchers neglect that the TCM constitution scale should be based on the feelings of the past year, which leads to confusion on TCM constitution and TCM syndrome.

4.3. Limitations of the Study. This study analyzes the distribution proportion of different TCM constitutions in IS patients, and the correlation among region, gender, and TCM constitution. However, it fails to study the correlation between TCM constitution and nationality, occupation, age, marital status, course of disease, biochemical index, TCM syndrome, and other factors in IS patients, as it lacks data report in the original literature. This, it is more difficult to figure out the factors affecting TCM constitution.

The results are likely to be biased due to the high heterogeneity of the cross-section studies in the meta-analysis, which is related to many factors of each study such as the region, sample size, diagnostic criteria, and measurement methods. However, TCM constitution theory believes that the differences in constitution are due to region and gender. And the features of TCM constitution are reflected in the results of the national norm [54]. Hence, to describe the overall TCM constitution distribution features of IS patients in China, this study analyzes the data of original literature comprehensively and categorizes them by region and gender for more accurate results. 


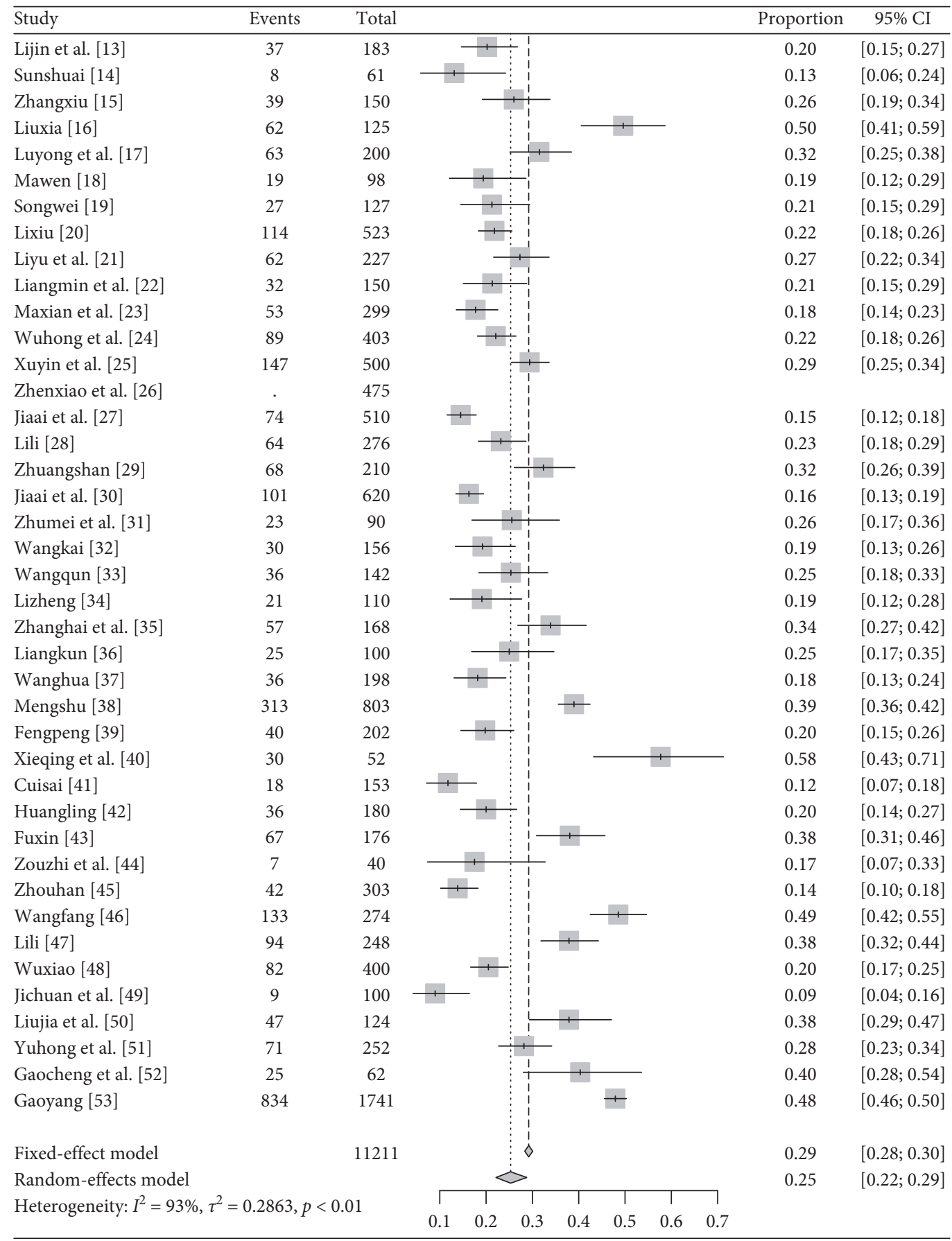

FIgURE 4: Meta-analysis of QDC's rate in IS patients.

4.4. Enlightenment to Clinical and Scientific Research. The results suggest that the groups most in need of IS prevention are qi-deficiency, phlegm-dampness, and blood stasis constitution. Some effective prevention measures are health education, lifestyle, and TCM intervention. The TCM constitution could be applied for the clinical treatment of ischemic stroke (QDC, PHC, BSC, and other types). The differentiated treatment is offered based on the TCM constitution, and the conformational effect between TCM constitution and syndrome.
In this study, the evidence-based medicine research method is adopted in the field of TCM constitution in IS patients for the first time, and the literature on the relationship between IS and TCM constitution is systematically evaluated and analyzed by meta-analysis. The research ideas and methods can be used as a reference for the literature review on the association between diseases and TCM constitution in the future. It is suggested that a scientific and feasible research plan should be formulated and published before implementing "body-disease related" clinical studies 


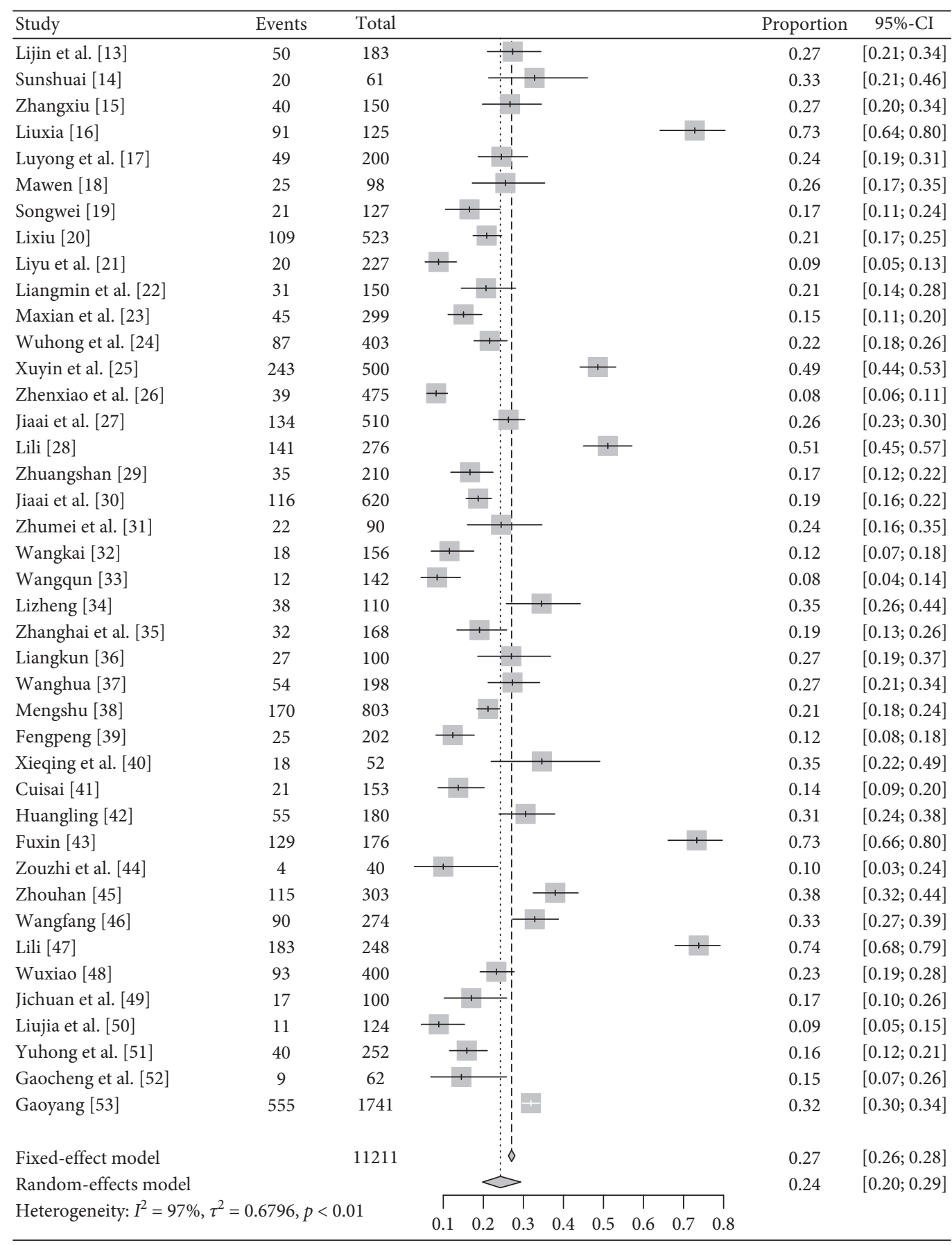

FIGURE 5: Meta-analysis of PDC's rate in IS patients.

(cross-sectional studies, case-control studies, and cohort studies). The process could improve the research plan quality though peer review. A general population control group could be organized for the cross-sectional study to compare the differences in TCM constitution between groups, so as to examine the TCM constitution closely related to the disease. As advised, a case-control study or prospective cohort study should be carried out for the possible high-risk TCM constitution of a disease. This is to identify the causal relationship between the TCM constitution and the disease, especially true for heavy chronic disease like stroke. For a thorough analysis of the factors influencing TCM constitution, interference factors shall be controlled in line with the international norms of observational research methodology of quality evaluation. This is to guarantee the accuracy of TCM constitution identification, and collect demographic disease information in detail. STROBE statement is recommended to make standardized reports when writing research papers [55], and thereby improving research quality and providing high-quality original evidence for systematic evaluation. 


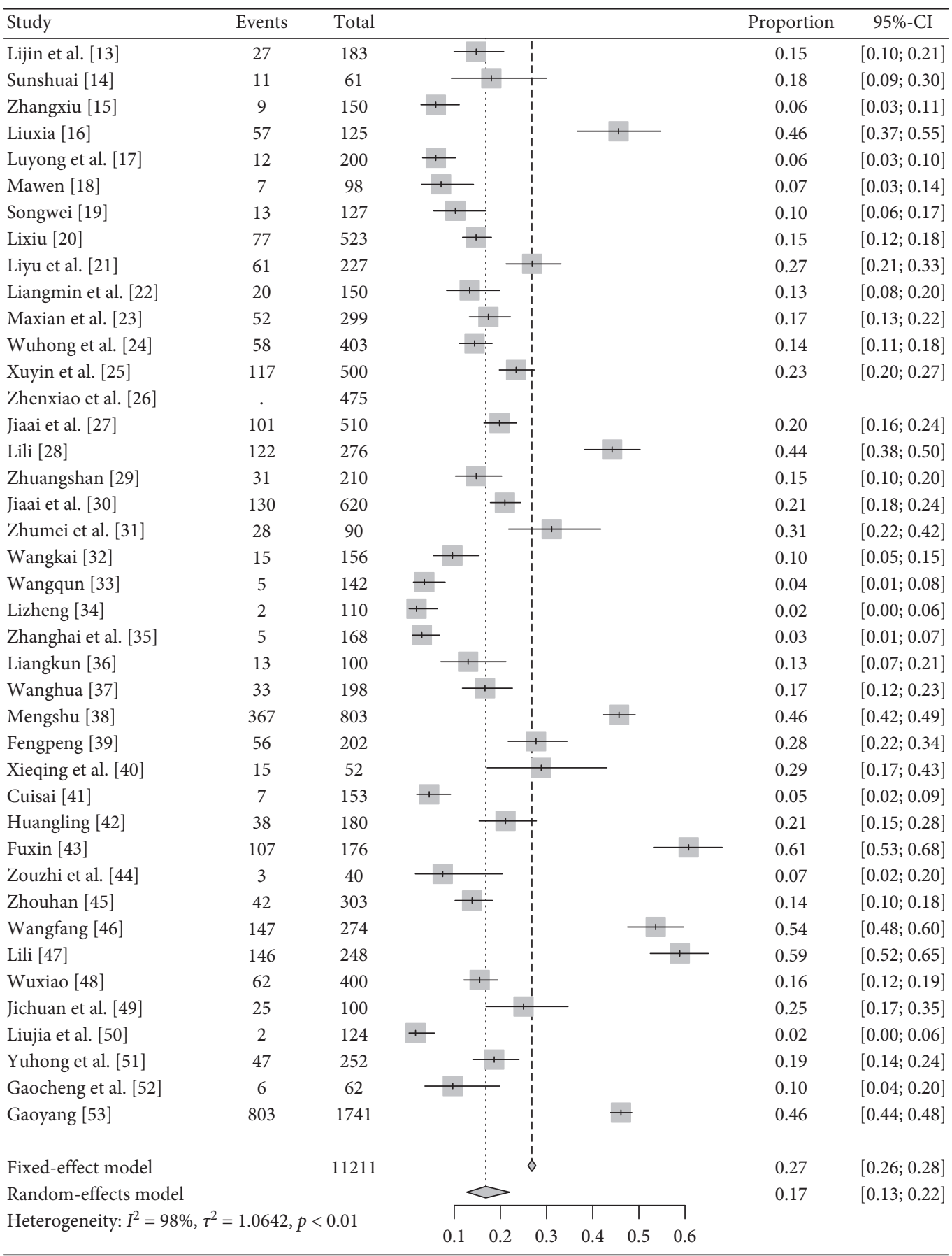

FIgURE 6: Meta-analysis of BSC's rate in IS patients.

TABle 3: Meta-analysis of rate of other TCM constitutions in IS patients.

\begin{tabular}{lcrr}
\hline Constitution type & Studies included & Proportion (\%) & 95\% CI \\
\hline Yin deficiency & 40 & 13 & {$[0.11,0.15]$} \\
Yang deficiency & 40 & 10 & {$[0.08,0.12]$} \\
Balance & 40 & 7 & {$[0.05,0.11]$} \\
Qi stagnation & 40 & 7 & {$[0.05,0.09]$} \\
Damp-heat & 40 & 6 & {$[0.05,0.07]$} \\
Inherited & 40 & 1 & {$[0.01,0.02]$} \\
\hline
\end{tabular}




\section{Conclusions}

The meta-analysis of 11,211 subjects finds that PDC, QDC, and BSC types are the main TCM constitutions in IS patients, QDC and PDC are risk factors for IS, and QDC is most closely related to IS. It is suggested that the future clinical observation research on the correlation between TCM constitutions and diseases should improve research quality, comprehensively explore the factors influencing TCM constitution, and thoroughly study the association between constitution and disease. Thereby, it could provide valuable reference for the clinical diagnosis and treatment of differentiated syndrome and diseases and adopt relevant implementation and reporting norms of evidence-based medicine.

\section{Conflicts of Interest}

The authors declare that there are no conflicts of interest regarding the publication of this paper.

\section{Acknowledgments}

This research was funded by National Key Research and Development Program (2018YFC1705000 and 2018YFC1705001).

\section{References}

[1] "Ischemic stroke," 2018, https://emedicine.medscape.com/ article/1916852-overview.

[2] D. Mozaffarian, E. J. Benjamin, A. S. Go et al., "Heart disease and stroke statistics--2015 update: a report from the American Heart Association," Circulation, vol. 131, no. 4, pp. e29-322, 2015.

[3] J. Adamson, A. Beswick, and S. Ebrahim, "Is stroke the most common cause of disability?" Journal of Stroke and Cerebrovascular Diseases, vol. 13, no. 4, pp. 171-177, 2004.

[4] V. L. Feigin, M. H. Forouzanfar, R. Krishnamurthi et al., "Global and regional burden of stroke during 1990-2010: findings from the global burden of disease study 2010," The Lancet, vol. 383, no. 9913, pp. 245-255, 2014.

[5] Stroke Association, "State of the nation: stroke statistics," 2018, https://ww.stroke.org.uk/system/files/sotn_2018.pdf.

[6] P. A. Wolf, R. B. D’Agostino, A. J. Belanger, and W. B. Kannel, "Probability of stroke: a risk profile from the Framingham study," Stroke, vol. 22, no. 3, pp. 312-318, 1991.

[7] J. Huang, Y. Cao, J. Guo et al., "Modified Framingham stroke profile in the prediction of the risk of stroke among Chinese," Chinese Journal of Cerebrovascular Diseases, vol. 10, no. 5, pp. 228-232, 2013.

[8] X. Li, J. Pang, M. Li, and D. Zhao, "Discover high-risk factor combinations using Bayesian network from cohort data of National Stoke Screening in China," BMC Medical Informatics and Decision Making, vol. 19, no. 2, p. 67, 2019.

[9] W. Qi, "Body constitution identification is the starting point of curing disease," Global Traditional Chinese Medicine, vol. 3, pp. 5-6, 2019.

[10] China Association of Chinese Medicine, Classification and Determination of TCM Constitution, China Press of TCM, China, 2009.
[11] H. L. Zeng, "Series four of meta-analysis:quality assessment tools for observational studies," Chinese Journal of EvidenceBased Medicine, vol. 4, no. 4, pp. 297-299, 2012.

[12] A. Stang, "Critical evaluation of the Newcastle-Ottawa scale for the assessment of the quality of nonrandomized studies in meta-analyses," European Journal of Epidemiology, vol. 25, no. 9, pp. 603-605, 2010.

[13] Lijin, "Analysis of TCM constitution type distribution and clinical characteristics in patients with ischemic stroke," New Journal of Traditional Chinese Medicine, vol. 11, 2017.

[14] Sunshuai, Study on the Correlation of Constitution, Syndromes, Inflammation-Related Proteins and MTHFR Gene Polymorphisms in Patients with Ischemic Stroke, China Academy of Chinese Medical Science, Beijing, China, 2017.

[15] Zhangxiu, Study on the Relationship between TCM Constitution and Physiological Risk Factors in Patients with Ischemic Stroke, Fujian University of Traditional Chinese Medicine, Fuzhou, China, 2017.

[16] Liuxia, Study on the Correlation between TCM Constitution and Short-Term Prognosis in Patients with Ischemic Stroke, Beijing University of Chinese Medicine, Beijing, China, 2016.

[17] L. Y. Cai, W. L. Dong, Q. S. Bin et al., "Distribution and related factors of TCM constitution types in patients with cerebral infarction in Shanghai Baoshan Youyi area," Modern Distance Education of Chinese Traditional Medicine, vol. 24, 2016.

[18] Mawen, Study on Syndrome Difference and Constitution Type of Left and Right Hemiplegia in Ischemic Stroke, Shandong Traditional Chinese Medicine University, Jinan, China, 2016.

[19] Songwei, Clinical Analysis of Supratentorial Watershed Infarction and Constitution of Traditional Chinese Medicine, Shandong Traditional Chinese Medicine University, Jinan, China, 2016.

[20] Lixiu, Analysis of Constitution and Risk Factors during Convalescence of Cerebral Infarction, Shandong Traditional Chinese Medicine University, Jinan, China, 2015.

[21] L. Y. Mie, S. Y. Han, and Y. Y. Ling, "Analysis of TCM constitution types in 178 senile ischemic apoplexy patients," Journal of Yunnan College of Traditional Chinese Medicine, vol. 2, 2015.

[22] L. M. Ying, J. Z. Long, and Y. S. Xian, "Distribution characteristics of TCM constitution types in 150 patients with ischemic stroke in Jiangmen area," New Journal of Traditional Chinese Medicine, vol. 2, no. 2, 2015.

[23] M. X. Jun, W. Hui, N. Qian et al., "Correlation analysis of progressive apoplexy and constitution type of traditional Chinese medicine," The Doctor of Traditional Chinese Medicine in Sichuan, vol. 2, 2015.

[24] W. H. Bin and Z. J. Wei, "Study on characteristics of constitution distribution of traditional Chinese medicine during convalescence of cerebral infarction," Journal of Emergency in Traditional Chinese Medicine, vol. 11, 2015.

[25] X. Y. Ping, Y. Chuan, and S. Bin, "Study on the distribution of TCM constitution in 500 patients with acute ischemic stroke in Pinggu District, Beijing," Beijing Traditional Chinese Medicine, vol. 1, 2015.

[26] Z. X. Min, C. Xi, G. D. Ying et al., "Study on the correlation between traditional Chinese medicine constitution and ischemic stroke," Journal of Emergency in Traditional Chinese Medicine, vol. 4, 2015.

[27] J. A. Ming, T. Jing, H. W. Mei et al., "The constitution factor and TCM inducement of recurrent ischemic stroke," Chinese Journal of Gerontology, vol. 9, 2014.

[28] Lili, Study on TCM Constitution, Risk Factors of Stroke and Carotid Arteriosclerosis in Patients with Cerebral Infarction, 
Traditional Chinese Medicine University Of Guangzhou, Guangzhou, China, 2014.

[29] Zhuangshan, Study on the Correlation between the Constitution of Traditional Chinese Medicine and Cerebral Atherosclerotic Stenosis in Anterior Circulation Cerebral Infarction, Shandong Traditional Chinese Medicine University, Jinan, China, 2014.

[30] J. A. Ming, H. W. Mei, Z. Hong et al., "Study on constitution and syndromes of 620 convalescent ischemic apoplexy patients," Chinese Journal of Information on Traditional Chinese Medicine, vol. 20, no. 6, pp. 14-16, 2013.

[31] Z. M. Zhen, S. C. Shou, and Q. Y. Jie, "Study on the correlation between constitution type and syndrome differentiation of ischemic stroke," in Proceedings of the 14th Chinese Medicine Diagnostic Academic Conference of China Association of Traditional Chinese Medicine, Chengdu, Sichuan, China, September 2013.

[32] Wangkai, Investigation and Analysis of TCM Constitution Types in 78 Patients with Cerebral Infarction, Hubei University of Chinese Medicine, Wuhan, China, 2012.

[33] Wangqun, Study on the Relationship between Syndrome Types and Constitution Classification of Acute Ischemic Stroke, Shandong Traditional Chinese Medicine University, Jinan, China, 2012.

[34] Lizheng, Study on the Correlation between Common Constitution Types and Interventionable Risk Factors of Ischemic Stroke, Henan University of Traditional Chinese Medicine, Zhengzhou, China, 2011.

[35] Z. H. Mei, Z. Y. Yun, and G. D. Ying, "Study on clinical characteristics of patients with different constitution types of ischemic stroke," Liaoning Journal of Traditional Chinese Medicine, vol. 8, pp. 1409-1412, 2010.

[36] Liangkun, Effect of TCM Constitution Type on Short-Term Prognosis of Cerebral Infarction with Atherosclerosis, Shandong Traditional Chinese Medicine University, Jinan, China, 2018.

[37] Wanghua, Clinical Study on TCM Constitution of Patients with Ischemic Apoplexy in Shijiazhuang Area, Hebei North University hebei North college, Zhangjiakou, China, 2018.

[38] Mengshu, Characteristics of TCM Syndromes and Constitution in Ischemic Stroke and Their Correlation with Compound Endpoint Events, Guangzhou University of Chinese Medicine, Guangzhou, China, 2019.

[39] Fengpeng, The Correlation between Blood Stasis in Patients with Ischemic Stroke and Risk Factors of Ischemic Stroke, Changchun University of Chinese Medicine, Changchun, China, 2019.

[40] X. Qing, G. D. Mei, and G. L. Hua, "Study on syndromes and constitution types of convalescent patients with ischemic stroke," Yunnan Journal of Traditional Chinese Medicine and Materia Medica, vol. 40, no. 9, pp. 36-38, 2019.

[41] Cuisai, Clinical Study on the Constitution of Traditional Chinese Medicine and the Etiological Classification of CISS and ApoE Polymorphism of Cerebral Infarction, Shandong Traditional Chinese Medicine University, Jinan, China, 2018.

[42] X. Qing, G. D. Mei, and G. L. Hua, Correlation Study of Constitution, Syndromes and APOE Gene Polymorphism in Patients with Acute Ischemic Stroke, Jiangxi University of Traditional Chinese Medicine, Nanchang, China, 2019.

[43] Fuxin, Correlation Analysis of TCD Changes and Constitution in Patients with Acute Ischemic Stroke, Changchun University of Chinese Medicine, Changchun, China, 2018.

[44] Z. Z. Qiang, Z. Z. Ying, Z. X. Xian et al., "Study on identification of lipoprotein $A$ and traditional Chinese medicine constitution in patients with acute cerebral infarction and high-risk population of stroke," China Practical Medicine, vol. 15, no. 23, pp. 143-146, 2020.

[45] Zhouhan, Study on the Correlation between Risk Factors of Acute Cerebral Infarction and Constitution of Traditional Chinese Medicine, Hunan University of Chinese Medicine, Changsha, China, 2019.

[46] Wangfang, Study on the Related Factors of Stroke Recurrence Based on the Theory of Cure for Disease, Beijing University of Chinese Medicine, Beijing, China, 2019.

[47] Lili, Study on the Correlation between MRI Lesion Location and Physique of Acute Cerebral Infarction Head Based on Association Rules, Changchun University of Chinese Medicine, Changchun, China, 2018.

[48] Wuxiao, Study on Secondary Prevention of Stroke Based on Healthy Rural Demonstration Community in Beijing and TCM Constitution Science, Beijing University of Chinese Medicine, Beijing, China, 2018.

[49] J. C. Rong, C. Li, L. L. Yuan et al., "Correlation between TCM constitution distribution and HCY in atherosclerotic apoplexy," Journal of China Prescription Drug, vol. 18, no. 7, pp. 151-152, 2020.

[50] J. Liu, F. Xu, N. Mohammadtursun, Y. Lv, Z. Tang, and J. Dong, "The analysis of constitutions of traditional Chinese medicine in relation to cerebral infarction in a Chinese sample," Journal of Alternative and Complementary Medicine, vol. 24, no. 5, pp. 458-462, 2018.

[51] Y. H. Juan, S. J. Long, and G. Ming, "Investigation and analysis of physique of 252 patients with cerebral infarction during convalescence," Practical Journal of Medicine \& Pharmacy, vol. 35, no. 2, pp. 152-154, 2010.

[52] G. C. Sen and R. X. Fei, "Distribution characteristics of TCM constitution in 120 patients with acute cerebral apoplexy," China Health Care \& Nutrition, vol. 28, no. 26, p. 361, 2018.

[53] Gaoyang, Multiple Evaluation of the Longitudinal Data Early Warning Model of Ischemic Stroke Recurrence with Combination of Disease and Syndrome, China Academy of Chinese Medical Science, Beijing, China, 2020.

[54] Z. Y. Bo, W. Qi, D. Q. Wei et al., "Epidemiological survey of TCM constitution in general population in China -- based on the data of 21,948 cases in 9 provinces and cities in China," Chinese Journal of Traditional Chinese Medicine, vol. 24, no. 1, pp. 7-12, 2009.

[55] E. Von Elm, D. G. Altman, M. Egger, S. J. Pocock, P. C. Gøtzsche, and J. P. Vandenbroucke, "The strengthening the reporting of observational studies in Epidemiology (STROBE) statement: guidelines for reporting observational studies," International Journal of Surgery, vol. 12, no. 12, pp. 1495-1499, 2014. 